\title{
Hacia una redefinición de las competencias y perfiles profesionales del comunicador audiovisual en el ecosistema digital
}

\section{Redefining competences and professional profiles of the audiovisual communicator in the digital ecosystem}
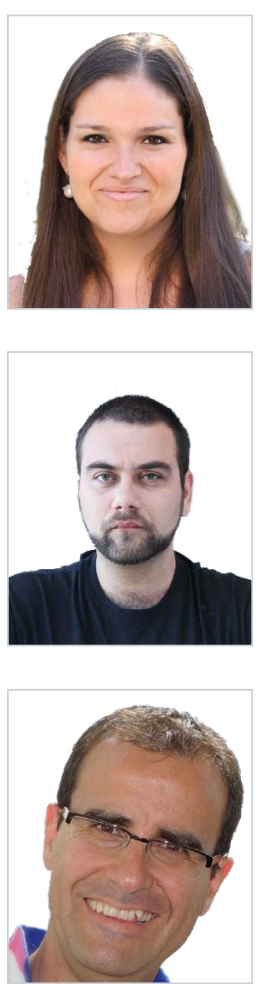

Marta Saavedra Llamas. Doctora en Ciencias de la Información por la UCM y desarrolló la tesis "La estrategia de comunicación en el cine de Pedro Almodóvar". Licenciada en Periodismo, realizó el postgrado Comunicación bursátil y sectores financieros. Ha trabajado como redactora en El faro de Murcia, El diario de Murcia, El nuevo Lunes, Dirigentes y Nuestros Negocios. De 2008 a 2010 fue redactora jefa de estas dos últimas publicaciones. Tiene experiencia en comunicación institucional y evaluación de la calidad universitaria; ha trabajado en ACAP. Dirige el Departamento de Comunicación de la Facultad de Comunicación y Artes de la Universidad Nebrija. Universidad Nebrija, Madrid, España msaavedr@nebrija.es

ORCID: 0000-0002-7762-9996

\begin{abstract}
Nicolás Grijalba de la Calle. Licenciado en Periodismo por la Universidad Nebrija, Máster de Práctica Escénica y Cultura Visual de la UCLM-Museo Reina Sofía y Doctor en Ciencias de la Información por la Universidad Complutense de Madrid con la tesis "La imagen de Madrid en el cine español". Director del Grado en Comunicación Audiovisual de la Universidad Nebrija. Miembro de la Asociación Española de Historiadores de Cine. Socio de APM. Experiencia en diferentes medios como TVE, Servimedia y La Gaceta (Cultura), así como en revistas de cultura underground. Coordinador de la Ruta Fílmica del festival de cine Filmadrid.

Universidad Nebrija, Madrid, España

ngrijalb@nebrija.es
\end{abstract}

ORCID: 0000-0002-6857-978X

Luis Miguel Pedrero Esteban. Licenciado en CC. de la Información (UPSA) y Doctor en Comunicación Audiovisual (UAB). Ha sido redactor en la Cadena SER, colaborador en COPE y Onda Cero, guionista en 40 Principales, Dial y M80, y consultor de Kiss FM. Catedrático de Estructura de la Comunicación (UPSA), profesor visitante en Birmingham City University, Universidad de Siena y Universidad de Lima, e invitado en la UNAM, la Universidad de Concepción (Chile) y la Universidade do Minho (Portugal). Miembro de honor de AERO y de la Sociedad Española de Periodística. Editor del digital Pantallas, Ondas, Frames y Bits.

Universidad Pontificia de Salamanca, Salamanca, España

Impedreroes@upsa.es

ORCID: 0000-0003-4949-2360

Cómo citar este artículo:

Saavedra Llamas, M.; Grijalba de la Calle, N.; Pedrero Esteban, L. M. (2018). Hacia una redefinición de las competencias y perfiles profesionales del comunicador audiovisual en el ecosistema digital. Doxa Comunicación, 27, pp. 369-385.

https://doi.org/10.31921/doxacom.n27a18 
Recibido: 02/11/2018 - Aceptado:12/11/2018

\section{Resumen:}

La transformación digital de la industria audiovisual y de sus lógicas de creación, producción, distribución y comercialización de contenidos ha generado la necesidad de nuevas competencias y perfiles profesionales cuya adquisición no se contempla todavía de forma expresa en los planes de estudio vigentes del Grado en Comunicación Audiovisual. Tras exponer el origen y evolución de esta titulación y los efectos de la convergencia tecnológica sobre este ecosistema, el artículo presenta los resultados de la entrevista a un grupo de discusión integrado por especialistas de reconocida trayectoria que, además, acreditan una amplia experiencia docente: sus reflexiones permiten identificar y describir los perfiles, las competencias y las áreas profesionales exigidos en un mercado donde la cualificación tecnológica no resulta suficiente sin el conocimiento de las bases narrativas sobre las que se construyen las historias.

\section{Palabras clave:}

Comunicación Audiovisual; Universidad; Competencias;

Destrezas y habilidades; Consumo digital
Received:02/11/2018 - Accepted: 12/11/2018

\section{Abstract:}

The digital transformation of the audiovisual industry, as well as changes in the creation, distribution and marketing of content, have resulted in the need for people with new skills and professional profiles. However, the acquisition of such individuals is still not specifically stated in the current curriculums of the Audiovisual Communications Degree. After presenting the inception and development of this degree programme, as well as the effects of the technological convergence on this ecosystem, this article presents the results of a discussion group interview composed of specialists with acknowledged professional experience who also have a substantial amount of confirmed teaching experience. Their reflections allow for the identification and description of profiles, competencies and professional areas demanded by the contemporary market in which technological qualifications are simply not enough without the knowledge of the narrative bases on which stories are built.

Keywords:

Audiovisual Communication; University; Degree; Competencies; Skills and abilities; Digital consumption.

\section{Introducción}

La normalización de Internet como vehículo de acceso a la información, el ocio y el entretenimiento audiovisual está reconfigurando las lógicas tradicionales de creación, producción y distribución de las empresas de comunicación, que han pasado de atender a unos pocos mercados con millones de receptores a facilitar un consumo personalizado e interactivo a millones de mercados individuales. Por efecto de la convergencia digital, espectadores y oyentes se han convertido hoy en usuarios que participan de intensivos intercambios con medios clásicos y emergentes a través de dispositivos y canales cada vez más próximos a sus espacios e intereses.

Este marco ha propiciado la resignificación de los medios, de sus mediaciones e incluso de sus mediadores, los especialistas que desarrollan las actividades de una industria en la que se han introducido nuevos procesos y rutinas cuya capacitación, a menudo ligada a desarrollos tecnológicos, quizá no garantizan los programas formativos universitarios que hasta ahora acreditaban la cualificación de los egresados. El impacto de la digitalización ha resultado especialmente determinante en el ámbito profesional de la Comunicación Audiovisual, un título de grado cuya orientación académica actual precisa de una revisión en materias, contenidos y, sobre todo, en las competencias comprometidas en los vigentes planes de estudio. 


\subsection{Evolución de los estudios en Comunicación Audiovisual: perfiles y competencias}

Los estudios universitarios en Comunicación Audiovisual se implantan en España en 1971, cuando el Ministerio de Educación y Ciencia aprueba las primeras Facultades de Ciencias de la Información en la Universidad Complutense de Madrid, la Universidad Autónoma de Barcelona y la Universidad de Navarra. Hasta entonces los referentes formativos en comunicación -aunque no de modo específico en el ámbito audiovisual- los representaban la Escuela de Periodismo de El Debate (1926) y la Escuela Oficial de Periodismo (1941), dependiente de la Delegación Nacional de Prensa y Propaganda. Tras ellas surgirían las temáticamente más cercanas Escuela Oficial de Cinematografía y la Escuela Oficial de Radiodifusión y Televisión (Jones, 1998).

En las nuevas facultades el área audiovisual no constituía una disciplina independiente, sino que formaba parte de una de las tres ramas de las Ciencias de la Información: Periodismo, Ciencias de la Imagen Visual y Auditiva y Publicidad y Relaciones Públicas. Sería la Ley Orgánica 11/1983 de 25 de agosto la que establecería el primer paso para la escisión de las disciplinas en tres carreras y el Real Decreto 1427/1991 de 30 de agosto el que autorizó, finalmente, la Licenciatura en Comunicación Audiovisual. A partir de 1993 diferentes universidades incorporan este plan de estudios a su oferta académica: es el caso de la Universidad de Valencia-Estudi General y la Pompeu Fabra, a las que se sumarían en 1994 la Universidad de Salamanca y la Ramón Llull, en 1997 la Universidad SEK, y en 1998 las universidades Nebrija, San Pablo CEU y Barcelona.

La formación en este ámbito afrontaría una nueva adaptación con la entrada en vigor en 2007 del Espacio Europeo de Educación Superior (EEES), que supuso el rediseño de la estructura de títulos en tres niveles (grado, máster, doctorado), la introducción del ECTS como unidad homogénea de carga lectiva y la reducción del periodo formativo de cinco a cuatro años. Con relación al audiovisual, las universidades hubieron de afrontar nuevos retos que -como señalan Alberich, Guarinos y Mañas (2009)- implicaban posicionar la institución universitaria como principal centro de capacitación profesional de la industria y contribuir así a la mejora de las condiciones del mercado laboral, favoreciendo la imbricación de la docencia y la investigación para respaldar académicamente esta disciplina.

En esta fase de construcción y remodelación de la oferta académica en Comunicación Audiovisual el Libro Blanco de los Títulos de Grado en Comunicación (ANECA, 2005: 227-228) definió cuatro categorías profesionales como resultantes de la adquisición del título: 1) director, guionista y realizador audiovisual; 2) productor y gestor audiovisual; 3) diseño de producción y posproducción visual y sonora; 4) investigador, docente y experto en estudios visuales. Este reducido abanico despertó algunas voces críticas que observaban un registro competencial pobre y poco actualizado con respecto a la realidad del sector (Bartolomé y Aiello, 2006). De hecho, la Mesa sectorial de formación para el empleo del sector audiovisual, promovida por el Barómetro de empleo de la ciudad de Madrid (2010), mantenía que todavía en plena era analógica el 44\% de los empleadores consultados advertía una necesidad de formación y que el $22 \%$ observaba una falta de profesionales preparados para los desafíos del momento.

La progresiva consolidación del entorno de producción y consumo digital ha agudizado esta falta de concordancia entre la capacitación académica y la exigencia laboral en este entorno. Besalú-Casademont, Schena y Sánchez-Sánchez (2017) explican que existe un gran abanico de opiniones sobre las competencias que debe adquirir un profesional de la Comunicación Audiovisual y por eso convendría situar el debate en si la universidad ha de introducir constantemente nuevas 
habilidades en función de la continua e irreversible transformación del medio o debe apostar por competencias generales y actitudinales que permitan a los futuros profesionales adaptarse a los cambios.

En cualquier caso, la oferta de estudios de Grado en Comunicación Audiovisual se ha multiplicado en España durante los últimos años. Según el Registro de Universidades, Centros y Títulos (RUCT) del Ministerio de Educación, Cultura y Deporte, en el curso académico 2018/19 se imparten 45 títulos ligados a este ámbito profesional:

Tabla 1: Oferta académica de estudios de grado en el ámbito audiovisual

\begin{tabular}{|l|l|}
\hline DENOMINACIÓN & UNIVERSIDAD \\
\hline Grado en Comunicación Audiovisual & $\begin{array}{l}\text { Extremadura, Illes Balears, Mondragón, Antonio de Nebrija, Autónoma } \\
\text { de Barcelona, CEU Cardenal Herrera, Carlos III de Madrid, Católica San } \\
\text { Antonio, Complutense de Madrid, A Coruña, Alcalá, Barcelona, Burgos, } \\
\text { Granada, Málaga, Murcia, Navarra, Santiago de Compostela, Sevilla, Vic- } \\
\text { Universidad Central de Catalunya, Vigo, País Vasco, Europea de Madrid, } \\
\text { Europea del Atlántico, Europea Miguel de Cervantes, Fernando Pessoa- } \\
\text { Canarias, Francisco de Vitoria, Internacional de Catalunya, Jaume I de } \\
\text { Castellón, Miguel Hernández de Elche, Pompeu Fabra, Pontificia Comillas, } \\
\text { Pontificia de Salamanca, Ramón Llull, Rey Juan Carlos, Rovira iVirgili, San } \\
\text { Jorge, CEU San Pablo, Valencia (Estudi General) y Politécnica de Valencia }\end{array}$ \\
\hline Grado en Cine y Medios Audiovisuales & Barcelona \\
\hline Grado en Cinematografía y Artes Audiovisuales & Rey Juan Carlos \\
\hline Grado en Comunicación Audiovisual y Multimedia & Europea de Madrid \\
\hline Grado en Comunicación y Creación Audiovisual & Salamanca \\
\hline Grado en Medios Audiovisuales & Pompeu Fabra \\
\hline
\end{tabular}

Fuente: Elaboración propia a partir de la información disponible en el RUCT.

El crecimiento de los títulos y, por tanto, de los alumnos y titulados parece responder a las dinámicas contemporáneas del audiovisual a nivel internacional: se vive un proceso de reconversión acelerado por el impacto de las tecnologías digitales, por el auge de los servicios de vídeo bajo demanda, el aumento sin precedentes del consumo y, al mismo tiempo, el cambio de hábitos y rutinas en el acceso al ocio y entretenimiento. En este sentido, la Unión Europea considera el sector audiovisual como un ámbito estratégico de crecimiento clave para el desarrollo económico, social y cultural, y mantiene desde 1991 el programa MEDIA -en 2014 se convertiría en subprograma de Europa Creativa- para impulsar la industria audiovisual de los países miembros (Creative Europa, 2018).

En España parece haberse consolidado la recuperación del sector iniciada en 2014: el Informe Económico Sectorial de las Telecomunicaciones y el Audiovisual publicado por la Comisión Nacional de los Mercados y la Competencia en 2017 recoge un crecimiento económico del 7,3\% en el último año, llegando a una facturación de 4.135 millones de euros. Estos datos se asientan sobre el crecimiento del $7 \%$ de los ingresos comerciales y sobre el aumento de ingresos por suscripción de contenidos audiovisuales en un 10,1\% (CNMC, 2017: 176-189). Con relación a este último indicador, conviene advertir que la penetración de la televisión de pago ya alcanza a casi la tercera parte de la población (28,5\%), una tendencia que, como 
se expondrá más adelante, resulta determinante en la reconfiguración de los procesos de creación y producción en este ámbito y, por ello, en las habilidades necesarias para desempeñar nuevas responsabilidades.

Nos hallamos, pues, ante un escenario de renovadas lógicas profesionales que sugiere la reformulación de las exigencias que deben afrontar hoy los egresados en Comunicación Audiovisual. El presente artículo se propone identificar cuáles son esos nuevos perfiles a partir de la descripción del entorno contemporáneo y de la experiencia de quienes ya se están enfrentando a los desafíos de la convergencia tecnológica y cultural: se intenta así determinar qué competencias se precisan para el actual ecosistema con el fin de que el catálogo sirva de base para próximas revisiones académicas sobre la planificación de los estudios universitarios de Grado en Comunicación Audiovisual. De forma concreta, los objetivos son:

- Describir la evolución y el estado contemporáneo de la industria audiovisual a nivel global y en España.

- Enunciar los hitos más destacados en la industria audiovisual como efecto de la digitalización.

- Reconocer el catálogo de perfiles profesionales que se demandan en la industria.

- Valorar si resulta efectiva la convivencia entre los perfiles clásicos y los nuevos roles profesionales.

- Sistematizar las competencias profesionales necesarias en este nuevo entorno.

- Identificar nuevos roles profesionales y tendencias de futuro.

La aceleración en la migración del sistema mediático al entorno digital y en los efectos profesionales de este cambio explican que los trabajos que hasta ahora habían analizado la formación superior en comunicación en España y las competencias que proporciona no describan los perfiles generados en el contemporáneo ecosistema de convergencia. Estos estudios presentan mayoritariamente un alcance generalista y referido a las disciplinas clásicas, sin acotar de forma específica los contenidos y materias de Comunicación Audiovisual.

A partir de la irrupción del Espacio Europeo de Educación Superior varias investigaciones abordaron la renovación de los estudios de Ciencias de la Comunicación desde una perspectiva general: entre otras, las de Sierra (2010), Sierra y Cabezuelo (2010), Vivar (2011), Ortiz Sobrino (2012) o Perlado y Rubio (2012). Más recientemente, Perlado y Saavedra (2017) analizaron los títulos oficiales de grado y máster en comunicación, incluidos los Grados en Comunicación Audiovisual, aunque el objetivo fue detectar el peso del área de la dirección de comunicación en los planes de estudios.

Civil i Serra (2018) revisaron la oferta de títulos en Comunicación al objeto de diseñar un plan estratégico con relación a la propuesta de programas. Miguel (2018) indagó en el abanico de títulos de Periodismo, Publicidad y RRPP y Comunicación Audiovisual para identificar las competencias digitales introducidas; su investigación confrontó las ofertas de empleo con la realidad competencial de los estudios atendiendo a las guías docentes de sus asignaturas, y dentro del área audiovisual destacó el desequilibrio entre los perfiles profesionales que se demandaban en la edición digital, producción y programación, y las habilidades potenciadas en la universidad. Otras áreas trasversales que tampoco se cubren con solvencia son la creatividad digital, el diseño digital y la gestión de comunidades.

En esa misma línea, Navío Navarro, González-Díez y Puebla-Martínez (2018) trataron de confirmar si los planes de estudios en comunicación forman en las competencias propias de los cada vez más solicitados roles de Community Manager y Social Media Strategist. Ligadas también a la escena audiovisual están las investigaciones de Heredero y Reyes (2016), quienes sostienen que se precisa de un profesional versátil, que controle todas las fases de creación y además pueda plasmar 
una idea en un proyecto para ser defendido ante posibles financiadores; debe dominar además la presencia en redes que utilizará para la promoción de su producto y la fidelización de su audiencia. Guarinos y Sedeño (2013), por último, concluyeron que el profesional debe controlar las fórmulas emergentes de construcción narrativa a partir de la transmedialidad del discurso.

\subsection{La industria audiovisual en el entorno digital}

La consolidación de la Red como ventana generalizada para el consumo de información y entretenimiento ha propiciado un nuevo escenario para el negocio de la comunicación en el que la industria se ha visto obligada a renovar sus rutinas analógicas, a redefinir sus tradicionales procesos de creación y comercialización de contenidos audiovisuales e incluso a reinventar sus modelos de negocio. En un entorno de consumo digital que se caracteriza por la atemporalidad, la ubicuidad, la conectividad, la multimodalidad y la interactividad en la relación entre creadores y usuarios (Siemens, 2008), se imponen hoy nuevas dinámicas en las ya no solo cobra relevancia el valor, originalidad o tratamiento del contenido, sino también su adaptabilidad a los nuevos dispositivos y soportes.

De hecho, el cambio más relevante y decisivo del ecosistema mediático contemporáneo lo constituye la consolidación del smartphone como dispositivo masivo para el consumo online. Aunque originalmente se concibió como un instrumento más o menos elitista y de apoyo a la gestión en ámbitos políticos y económicos (Canavilhas, 2013), el móvil se ha extendido con tal contundencia a escala mundial que las cifras en torno a su posesión y uso determinan la planificación y las estrategias de comunicación a nivel informativo, persuasivo y comercial. Según el informe Tráfico Global de Datos Móviles 20162021, en 2021 habrá en todo el planeta más teléfonos móviles (5.500 millones) que suministros de agua corriente (5.300 millones) y cuentas bancarias (5.400 millones), y el incremento exponencial de los usuarios, smartphones y conexiones de Internet en el hogar (Internet of Things, IoT) hará que el intercambio de datos móviles pase a representar el $20 \%$ del tráfico IP frente al 8\% de 2016 (Cisco VNI Mobile, 2017).

La omnipresencia del móvil adquiere especial relevancia en nuestro país, que en 2016 se convirtió -junto a Singapur- en el que contaba con más terminales inteligentes por habitante del mundo: un 92\% de sus ciudadanos disponía de al menos uno de estos dispositivos, un 4\% más que en 2015 (AEDE, 2016). Semejantes cifras explican que la mitad de los adolescentes españoles de entre 15 y 24 años ya consuman entre el 90 y el 100\% de su tiempo en red sobre la pantalla móvil, según el informe La Sociedad Digital en España 2017 (Fundación Telefónica, 2017). Ese estudio revela también cómo el uso de aplicaciones de mensajería ha crecido en el último año un 394\% dado el incremento en el acceso a servicios que fusionan la comunicación con el entretenimiento (YouTube, Netflix, Instagram o Facebook, entre otras).

La mediatización de los móviles -el hábito del mobile first-está alterando, por un lado, la construcción de los tradicionales géneros discursivos: información en sus diferentes variantes, ficción, música y publicidad (Fernández, 2014); pero, además, está incidiendo en las pautas de difusión, cada vez más orientadas a una concepción multiplataforma en un intento por alcanzar a los usuarios digitales alejados de los receptores analógicos; por ejemplo, las cadenas de radio apuestan por la cobertura de noticias mediante la emisión simultánea por antena, web, redes sociales y alertas a móvil (Orrantia, 2018). El nuevo ecosistema comunicativo evidencia, en suma, la convergencia de fórmulas periodísticas tradicionales y las propias 
de los medios electrónicos, y la tecnología digital brinda así un horizonte de opciones comunicativas inimaginable no hace tanto tiempo a partir de una codificación numérica de las señales con la que se refuerza la virtualidad de imagen y sonido (Bandrés et al., 2004).

La progresiva migración al consumo de contenido audiovisual en dispositivos digitales no se puede considerar masiva, pues aún cohabitan dos rangos de audiencia claramente diferenciados: quienes emplean de forma activa las nuevas extensiones tecnológicas, a veces sujetas a ciclos de vida muy cortos, y quienes mantienen soluciones en declive o en vías de extinción. "El primero se ha universalizado en el público más joven y describe esos usos tecnológicos como señas de identidad generacional que definen tendencias de futuro" (Díaz Nosty, 2017: 33).

En cualquier caso, la aparición de nuevas plataformas, soportes y canales ha incrementado significativamente el tiempo de exposición a una oferta cada vez más amplia y variada: de los 354 minutos diarios que dedicábamos a los medios en 1997 (5 horas y 54 minutos) pasamos a 388 diez años después (6 horas y 27 minutos), lo que supone un incremento del 9,3\%; pero es que en los nueve años siguientes, en 2016, se experimentó una subida del 15,3\% hasta alcanzar los 447 minutos (7 horas y 27 minutos) en 2016.

Tabla 2: Tiempo medio de consumo diario de medios 1997-2016 (en minutos)

\begin{tabular}{|l|l|l|l|l|l|l|l|}
\hline & Diarios & Revistas & Radio & TV & Internet & Cine & Total \\
\hline 1997 & 15,0 & 5,7 & 100,2 & 231,2 & - & - & 354,1 \\
\hline 2007 & 16,8 & 3,1 & 111,6 & 222,1 & 31,8 & 1,3 & 388,1 \\
\hline 2016 & 9,5 & 2,1 & 103,6 & 223,1 & 107,7 & 0,7 & 447,3 \\
\hline
\end{tabular}

Fuente: Diez años que cambiaron los medios, Fundación Telefónica.

Si ese tiempo de atención mediática se extiende a lo largo de un año, alcanzaría un total de 113 días (cerca de cuatro meses) que, proyectados sobre una vida de 85 años, supone que un individuo con expectativas normales de existencia se expondría durante más de 26 a los medios. Esta cifra evidencia la creciente inmersión ciudadana en el audiovisual y el empuje de una industria cuya actividad podría llegar a acaparar en 2025 hasta 600 minutos de atención diaria.

Buena parte de este incremento en el tiempo de consumo mediático se explica por la irrupción en el mercado español de los servicios OTT (Over The Top) como Netflix, Amazon o HBO, cuya oferta de contenidos propios y cuotas de suscripción asequibles han multiplicado la cifra de abonados a estas plataformas. Según el Panel de Hogares recogido por la Comisión Nacional de los Mercados (CNMC) en el cuarto trimestre de 2017, los españoles ya dedican casi el mismo tiempo a ver contenidos audiovisuales en la tele que en otras pantallas (smartphone, tableta y ordenador): una media de tres horas diarias en el receptor tradicional frente a dos horas y media en dispositivos portátiles. La relación se invierte en la franja de 16 a 24 años: aquí el consumo conjunto en ordenador, tableta y móvil se sitúa en 4,7 horas, más del doble $(2,2)$ del dedicado la televisión.

El mercado de las plataformas audiovisuales de streaming se aventura como el principal motor de la industria a nivel mundial en los próximos años: según la empresa británica Digital TV Research Ltd, el número de abonados a los servicios de vídeo bajo demanda va a aumentar en 409 millones hasta 2023, cuando los clientes superarán los 775 millones a escala 
glogal (El Periódico, 2018). Considerando la inversión anual de estas compañías en ficción propia -solo Netflix ha comprometido 8.000 millones de dólares en 2018 (El Economista, 2018)-, su estrategia de producción local y la previsión de que la Comisión Europea imponga una cuota mínima del 30\% de contenidos europeos, se vislumbra un horizonte laboral permeable a los actuales y futuros profesionales de la Comunicación Audiovisual para desempeños y tareas cada vez más especializadas.

Un destino con parecidas oportunidades auspicia también la industria del audio, donde el monopolio que a lo largo de casi un siglo había protagonizado la radio hertziana se ha quebrado con la irrupción en el entorno digital de las emisoras nativas online (según el Primer Directorio Certificado de la Radio Online en España publicado en 2017 por la Asociación Española de Radio Online ya operan más de mil entidades), las plataformas de música automatizada (un mercado que en los primeros seis meses de 2018 sumó a nivel mundial 229'5 millones de suscriptores con una recaudación de 3.500 millones de dólares (IFPI, 2018)-, los creadores y agregadores de podcasts-industria que en 2017 alcanzó en EE UU 314 millones de ingresos, un $86 \%$ más que el año anterior (IAB, 2018)-y de los audiolibros, cuyas ventas crecieron a escala global un $22,7 \%$ en 2017 hasta alcanzar 2,5 billones de dólares.

Se han multiplicado las opciones de consumo sonoro, pero también las exigencias para su diseño y distribución: los dispositivos digitales -en su mayoría pantallas- favorecen la creación de nuevos lenguajes y narrativas (en estos soportes el audio primero se ve y luego se escucha) para atraer la atención de una audiencia juvenil acostumbrada a la interacción con el proveedor, al intercambio con sus iguales, al disfrute crossmedia y las experiencias transmedia, hábitos que hoy determinan la conceptualización, el desarrollo tecnológico y la comercialización de los productos comunicativos (Pedrero y Herrera, 2017).

Esa apuesta por proyectar las historias que emergen en un canal y se ramifican por otros adyacentes (televisión, radio, podcast, música, cine, videojuegos, cómic, redes sociales) evidencia la gran transformación en el acceso y consumo de contenidos audiovisuales instaurada en el actual entorno digital, así como las implicaciones que semejante cambio supone a nivel cultural, sociológico, económico y también educativo. Con el ya descrito contexto de macrodatos, algoritmos y aprendizaje robótico, de producción transmedia y difusión multi-soporte, las competencias de un comunicador audiovisual no terminan ya en el proceso creativo o productivo (objeto hasta ahora preferente de la capacitación académica), sino que se amplían hacia facetas y ámbitos de especialización con nuevas y constantemente renovadas competencias.

\section{Metodología}

La primera parte de esta investigación se asienta sobre un estudio descriptivo basado en fuentes secundarias que ayudan a contextualizar el fenómeno estudiado y a reconocer las claves que explican su transformación. Las referencias se han utilizado, por un lado, para explicar la evolución de los estudios en Comunicación Audiovisual y, por otro, para delimitar la situación de la industria audiovisual y su adecuación al entorno digital.

A continuación, se plantea una investigación de tipo cualitativo ad hoc concretada en un grupo de discusión; con esta metodología se consigue la expresión de distintas posturas y actitudes de los participantes, el intercambio de información y la orientación del discurso sobre la realidad a investigar (Canales y Peinado, 1995). Esta técnica ha servido para explorar 
los perfiles profesionales en Comunicación Audiovisual generados en el actual ecosistema, para revisar su estado y advertir tendencias de futuro.

La conformación del grupo de discusión se articuló sobre dos variables concurrentes: se seleccionaron profesionales en activo del ámbito audiovisual con una trayectoria igual o superior a ocho años y una experiencia docente no inferior a cuatro años en programas de formación relacionados con la comunicación; en el primer caso se consideró relevante la antigüedad para reconocer cómo ha evolucionado el mercado con el despliegue digital y poder así comparar los dos escenarios; y en el segundo caso se pretendía garantizar un acercamiento entre profesión y academia para que los expertos convocados dominasen los conceptos de 'perfil profesional' y 'competencias generales y específicas', y además conociesen la naturaleza de las titulaciones que se ofertan en la universidad española.

Además, se procuró que entre los participantes en el grupo estuvieran representados diferentes roles profesionales para crear áreas de desarrollo profesional en el campo audiovisual y garantizar que la mayoría de los perfiles estuvieran integrados. Cabe indicar que la muestra cualitativa no perseguía una representatividad estadística, sino estructural, pues el objetivo del estudio es comprender el sentido social y las relaciones con el fenómeno investigado, no la extrapolación de los datos al universo, como en el caso de la muestra cuantitativa (Rubio y Perlado, 2015). Dado que el grupo de discusión estuvo constituido por expertos, presentamos aquí un resumen de los participantes:

Tabla 3: Expertos que conforman el grupo de discusión

\begin{tabular}{|c|c|c|c|c|c|c|}
\hline Ámbito & Nombre & Perfil profesional & $\begin{array}{l}\text { Años } \\
\text { exp. prof }\end{array}$ & Área docente & $\begin{array}{l}\text { Años } \\
\text { exp. doc }\end{array}$ & Código \\
\hline Guion & Itziar Bernaola & $\begin{array}{l}\text { Guionista y directora } \\
\text { de informativos y series } \\
\text { documentales }\end{array}$ & 12 & Guion no ficción & 10 & E1 \\
\hline Dirección / Realización & Adrián Blanco & $\begin{array}{l}\text { Realizador series y películas } \\
\text { (Siete vidas, Buscando el } \\
\text { norte...) }\end{array}$ & 19 & Realización & 7 & E2 \\
\hline Producción & Rocío Gago & $\begin{array}{l}\text { Productora de series (Vis a } \\
\text { Vis, Los hombres de Paco...) }\end{array}$ & 17 & Producción & 5 & E3 \\
\hline Dirección artística & Saida Santana & $\begin{array}{l}\text { Directora artística de } \\
\text { proyectos audiovisuales y } \\
\text { actriz }\end{array}$ & 15 & $\begin{array}{l}\text { Dirección artística y } \\
\text { creativa }\end{array}$ & 9 & E4 \\
\hline Fotografía & $\begin{array}{l}\text { Amaya } \\
\text { Hernández }\end{array}$ & Artista visual y fotógrafa & 8 & $\begin{array}{l}\text { Dirección de } \\
\text { fotografía }\end{array}$ & 5 & E5 \\
\hline Edición & $\begin{array}{l}\text { Jesús M. } \\
\text { González }\end{array}$ & Editor & 11 & Edición y montaje & 9 & E6 \\
\hline Posproducción & Alejandro Priego & Posproductor & 8 & Posproducción & 5 & E7 \\
\hline Sonido & Sergio Testón & $\begin{array}{l}\text { Posproductor de sonido. } \\
\text { Remezclador }\end{array}$ & 11 & $\begin{array}{l}\text { Posproducción de } \\
\text { sonido }\end{array}$ & 4 & E8 \\
\hline
\end{tabular}




\begin{tabular}{|c|c|c|c|c|c|c|}
\hline Animación digital y 3D & Gonzalo Martín & $\begin{array}{l}\text { Experto en 3D y realidad } \\
\text { virtual }\end{array}$ & 15 & $\begin{array}{l}\text { Edición y modelaje } \\
\text { 3D }\end{array}$ & 11 & E9 \\
\hline Narrativa transmedia & Miguel Oliveros & Artista visual y videocreador & 8 & Nuevas narrativas & 5 & E10 \\
\hline Contenidos multimedia & Francisco Sierra & $\begin{array}{l}\text { Director Área Multimedia } \\
\text { Antena } 3\end{array}$ & 25 & $\begin{array}{l}\text { Comunicación } \\
\text { Digital }\end{array}$ & 10 & E11 \\
\hline Comunidades virtuales & Antonio García & $\begin{array}{l}\text { Coordinador social media } \\
\text { Atresmedia }\end{array}$ & 10 & $\begin{array}{l}\text { Gestión de redes } \\
\text { sociales }\end{array}$ & 4 & E12 \\
\hline Podcasting & $\begin{array}{l}\text { Ma Jesús } \\
\text { Espinosa }\end{array}$ & $\begin{array}{l}\text { Jefa de Proyecto Podium } \\
\text { Podcast (Prisa Radio) }\end{array}$ & 11 & $\begin{array}{l}\text { Innovación } \\
\text { radiofónica }\end{array}$ & 4 & E13 \\
\hline \multicolumn{7}{|c|}{ Fuente: Elaboración propia. } \\
\hline \multicolumn{7}{|c|}{$\begin{array}{l}\text { - PIl: ¿Qué nuevos perfiles profesionales, después de la implantación de lo digital, se han constituido en el ámbito de la } \\
\text { comunicación audiovisual? ¿Conviven estos nuevos perfiles con los tradicionales? } \\
\text { - PI2: ¿Qué competencias se adscriben a estos nuevos perfiles profesionales? } \\
\text { - PI3: ¿Qué nuevas tareas se incorporan a esos perfiles profesionales? ¿Qué áreas novedosas se han implantado o cree } \\
\text { que surgirán? }\end{array}$} \\
\hline
\end{tabular}

\section{Resultados}

\subsection{Redefinición de los perfiles profesionales}

Prácticamente todos los expertos entrevistados en esta investigación coinciden en señalar que las nuevas oportunidades profesionales del sector audiovisual están vinculadas de forma directa con el escenario digital; aseguran que las tecnologías de producción y de distribución ya permiten la multiplicación de los contenidos y que estos se diseñan pensando en una implicación mayor del espectador.

Los expertos identificaron distintos nuevos perfiles profesionales surgidos tras la consolidación del entorno digital y apuntaron tres roles muy concretos:

- Diseñadores, programadores y desarrolladores de aplicaciones y softwares

- Especialistas en marketing digital y en nuevas plataformas audiovisuales

- Creadores de escenarios virtuales y diseñadores 3D

Según su experiencia, el ecosistema audiovisual precisa de profesionales que conozcan a la perfección los nuevos parámetros digitales; que procuren un constante reciclaje en el aspecto tecnológico, pero que al mismo tiempo comprendan los intereses del consumidor y espectador actual. Como curiosidad resulta relevante apuntar que cerca de la mitad de los expertos no utilizan ya el término 'espectador' para referirse a la audiencia; ahora el protagonista es el usuario: se trata de un personaje activo que participa de forma definitiva en la construcción de los relatos propuestos. 
Se constata el consenso en admitir que esta narrativa inmersiva necesita para su desarrollo de profesionales creativos, interesados en una tecnología más humana, capaces de darle profundidad a la realidad virtual, los escenarios $360^{\circ}$ y las técnicas 3D más avanzadas:

“Las llamadas imágenes 'reales' tienen los días contados. Como mucho, sabrán relacionarse con los miles de imágenes virtuales que surgirán de los programas 3D. Gracias a esto, precisamente en la industria del audiovisual y en el escenario artístico han surgido diseñadores 3D, animadores 3D, iluminadores 3D y VFX, expertos en rigging y character fx... Gente muy cualificada capaz de dedicarle horas y horas a conseguir un movimiento o una textura concreta en un personaje” (E6).

Los expertos que provienen de ámbitos más técnicos asumen natural la incorporación de programadores y desarrolladores de aplicaciones en los proyectos de carácter audiovisual. Estos perfiles no pueden desligarse del aspecto creativo y deben combinar sus saberes puramente informáticos con un conocimiento profundo de los recursos narrativos, como expresa el E10. Por su parte, el E7 sostiene:

"El perfil del programador ya resulta más que necesario en la industria del videojuego: guionistas, diseñadores, programadores... trabajan mano a mano. Sin embargo, cada vez alcanza más importancia la figura del game designer en las redacciones de televisión y también en productoras que lideran proyectos complejos y de largo alcance".

Los profesionales vinculados con los grandes grupos de comunicación también aluden a la aparición en el sector audiovisual de roles adscritos al marketing digital: expertos del renovado negocio audiovisual que entienden la pertinencia de diferentes fórmulas para llegar a un público hiperconectado; son los encargados de elaborar, junto a figuras como el Community Manager o el Social Media Strategist, estrategias eficientes para las redes sociales y/o las distintas plataformas digitales. Así lo razona el E5:

"Las universidades necesitarán incorporar a sus planes asignaturas que doten al futuro profesional de conocimientos sobre la gestión del negocio audiovisual. El espíritu romántico del creador no debe chocar con las estrategias nacidas del marketing digital: si lo que queremos es que nuestra obra se vea y que llegue a determinado público, es imprescindible conocer los comportamientos del espectador, los caprichos de la industria".

Los entrevistados coinciden también en atribuir protagonismo a los profesionales de la edición y la postproducción: aunque son roles consolidados en la industria audiovisual, el incremento de los contenidos y las nuevas narrativas pensadas para smartphones y otros dispositivos portátiles requieren de más profesionales formados en estas áreas.

La reflexión sobre la redefinición o renovación de los perfiles profesionales se cerró con otra oportuna pregunta: ¿conviven los roles asociados al entorno digital con los que ya existían en el entorno analógico? La mayor parte de los expertos coincidieron en afirmar que sí, que las categorías profesionales tradicionales siguen existiendo, sólo que ahora necesitan una reorientación directa hacia el escenario digital:

"Pensemos en la figura del guionista: esta profesión que no se va a agotar. Sin embargo, el guionista hoy necesita ser mucho más versátil, más rápido que antes; necesita comprender que su historia puede lanzarse a todo el mundo, que ya no tiene 70 minutos para desarrollarla, tan sólo cuenta con $20 \ldots$ O que su proyecto ya no lo financia una televisión, sino una empresa láctea o una empresa energética" (E5). 


\subsection{Redefinición de competencias, habilidades y destrezas}

Los nuevos perfiles profesionales conducen hacia un nuevo desarrollo de competencias: además de apuntar las capacidades y aptitudes que debe atesorar en el actual ecosistema el comunicador audiovisual, los expertos advirtieron de la importancia que las empresas conceden en la actualidad a esa cualificación, al margen de si se proporciona en los planes de estudio vigentes con arreglo al Libro Blanco de los Títulos de Grado en Comunicación. En él las competencias se agrupan en cuatro grandes bloques: disciplinares, profesionales, académicas y específicas, y los expertos coinciden en señalar que son las profesionales y las específicas las que más se demandan en la actualidad.

El trabajo práctico, el dominio de las herramientas, la actualización permanente y una visión transversal del negocio audiovisual son los valores más asociados a estos nuevos perfiles. Sobre las competencias profesionales, los encuestados señalan la importancia de conocer los programas y herramientas para la narrativa inmersiva y la participativa, con especial atención a los proyectos que trabajan entornos virtuales y espacios imaginarios 3D. Estas técnicas no constituyen ya un "adorno caprichoso" de determinados cineastas, sino que han pasado a formar parte de la denominada "nueva comunicación sensorial”. El entrevistado E10 confirma que el modelado 3D se usa con frecuencia en la realidad aumentada para televisión (para crear, por ejemplo, simulaciones de hologramas), y apunta también a la presencia de estas herramientas para actos de comunicación política y eventos de toda índole. Lo mismo ocurre con el lenguaje de la programación y con la consolidación evidente de destrezas asociadas al ámbito del grafismo:

"A casi todos mis compañeros de proyectos ya se les presupone capacidad para contar historias, pero además han de demostrar conocimientos sólidos de programación creativa, diseño con entornos gráficos inmersivos e interactivos, editores y especialistas en la postproducción que sepan elevar a una nueva dimensión el audio y el vídeo. Por eso trabajamos tan unidos guionistas, artistas, productores, ingenieros" (E11).

También resulta indispensable que los nuevos roles entiendan y dominen los mecanismos propios de la comunicación digital. Varios expertos coinciden en apuntar que las competencias asociadas a la producción y distribución de contenidos en los medios digitales, y más concretamente en las redes sociales, no deben vincularse exclusivamente a los profesionales del periodismo y/o la publicidad, sino que la gente del audiovisual debe posicionarse para liderar también algunos perfiles como el de Social Media Analyst, el Community Manager, el Chief Blogging Officer y el Search Engine Optimizer (SEO):

"La televisión ha multiplicado su poder gracias a la aparición de las redes sociales, a las plataformas anexas y a los canales alternativos de consumo audiovisual en la Red, por ejemplo. Por eso, en las redacciones cada vez se contrata a más gente con amplitud de miras, versátiles y transversales: que dominen estos códigos” (E1).

Dos de los participantes, por su vinculación con el área de sonido, consideran que el sector audiovisual todavía está huérfano de expertos creativos en audio: se han de incorporar profesionales capaces de desarrollar nuevos productos radiofónicos aprovechando la fuerza de la emisión digital, ampliando sus posibilidades narrativas y comerciales. En este sentido, la emergencia del podcast como formato de consumo a la carta, llamado a replicar el hábito practicado con las plataformas de vídeo, reclama perfiles profesionales cada vez más especializados:

“Creo que los más demandados tendrán que ver con características concretas: Project Managers, Storytellers, diseñadores de ambientes sonoros, expertos en branded content, desarrolladores, programadores de apps y expertos en analítica 
y experiencia de usuario. Los perfiles más estáticos que no posean versatilidad y conocimiento de estas áreas (ideación, producción, desarrollo, venta y difusión) van a tener más complicada su llegada al mercado laboral” (E13).

Tras la exposición de este bloque de competencias profesionales, los expertos afirman con llamativa unanimidad que las empresas se interesan cada día más en destacar aquellos perfiles con una formación profunda y puramente humanística; se valoran especialmente los conocimientos culturales generales, dando por supuesto el dominio de las narrativas clásicas, así como una capacitación sólida en el campo de la composición visual y estética. Resulta significativo cómo para estos entrevistados es necesario acompasar el dominio de las nuevas tecnologías y la comprensión de las nuevas fórmulas narrativas con una robusta formación en otros saberes humanos de carácter artístico-filosófico:

"Cualquier profesional de la comunicación ha de saber y tener claro qué quiere contar y cómo lo quiere contar, asumiendo que la honestidad en el relato es siempre primordial. La era digital permite que todos participemos en la construcción de historias, pero no todo el mundo sabe tocar la tecla exacta ni todos aportan datos exactos para generar un contenido creativo nuevo" (E4).

"Es muy importante la creación y la existencia de la propia marca digital del profesional del audiovisual; saber que tiene unas actitudes y aptitudes que van más allá de su trabajo profesional hace que se consoliden entornos digitales y autorales muy personales" (E12).

En definitiva, los expertos coinciden en sugerir la reformulación de los planes de estudio de Comunicación Audiovisual para encontrar un equilibrio que permita a los estudiantes adquirir nuevas competencias profesionales -más que necesarias para el desarrollo de la profesión- a la vez que mantener e incluso recuperar contenidos y saberes básicos propios de las Humanidades.

\subsection{Transformación de las áreas profesionales}

Para finalizar, y en respuesta al tercer ámbito de investigación, los participantes en el grupo de discusión evaluaron la transformación que las áreas profesionales del sector audiovisual han sufrido desde la implantación del modelo digital y discutieron acerca de la consolidación de las nuevas tareas incorporadas a este ámbito. Conviene advertir que de entre todos los puntos tratados, éste ha sido el que suscitó más discrepancias, pues si bien se reconoce que las nuevas áreas profesionales han surgido ligadas a la consolidación de las redes sociales y a los nuevos modelos de negocio (en especial el de las plataformas VOD), los expertos valoran esta situación con prudencia -todavía no se han consolidado- y dependiendo del área de especialización del que proceden.

En cualquier caso, las nuevas oportunidades profesionales parecen estar relacionadas con el cambio de hábitos en el acceso y el consumo de contenidos, lo que conduce a la revisión de unos modelos de negocio que deben basarse en tres principios básicos: la versatilidad, la innovación y la transversalidad. Por ello, y frente al dominio de las nuevas herramientas digitales, son cada vez más los expertos que demandan un trabajo práctico basado en los valores de la calidad, la creatividad, la veracidad y la independencia:

"Entre las tareas que se requerirán a estos nuevos perfiles están las vinculadas al dominio de amplias destrezas expresivas y técnicas: personas capaces de desarrollar habilidades personales y profesionales propias del mundo global, excesivamente dinámico y en perpetuo cambio [...] Pero no podemos olvidar que estos profesionales deberán reforzar también sus conocimientos teóricos, adquirir un bagaje cultural importante y trabajar capacidades de liderazgo y empatía” (E8). 


\section{Conclusiones}

La transformación digital en la que se halla inmersa la industria audiovisual ha generado un nuevo escenario de exigencias y retos profesionales sobre los que se están redefiniendo las lógicas de creación y producción, los canales de distribución, los modelos de negocio y hasta la naturaleza de los receptores, cuya condición de espectadores y oyentes pasivos en el entorno analógico se ha convertido en la de usuarios que consumen de forma activa las propuestas de ocio y entretenimiento del ecosistema contemporáneo. La incidencia de la tecnología en los procesos de generación y distribución de contenidos determina la cualificación requerida por los agentes que participan en este entorno: la hibridación de lenguajes, la mediatización de los móviles, la inmediatez en el acceso y la personalización del contenido han propiciado la emergencia de perfiles con polivalentes competencias y destrezas.

Los expertos consultados para esta investigación coinciden en destacar las oportunidades que implica la adaptación al modelo digital y la consiguiente reorientación de los roles en la industria: hacen falta profesionales capaces de diseñar relatos con una visión global, en los que se conjuguen las técnicas propias del desarrollo narrativo con las posibilidades de adecuación multisoporte y la proyección comercial hacia nuevos modelos de negocio. La evolución de los indicadores sobre penetración, crecimiento y gasto de la industria audiovisual, así como los datos de consumo de contenidos, exposición mediática y acceso a nuevas plataformas y servicios de vídeo bajo demanda, vislumbran un horizonte laboral lleno de oportunidades para los actuales y futuros profesionales de este sector.

Aunque el escenario digital plantea mayores dosis de ingenio y tecnificación, sigue siendo obligado el compromiso del creador con los valores esenciales a todo relato: historias sugerentes, bien definidas, que faciliten oportunas claves de interpretación y ayuden a la comprensión de nuestro entorno social y cultural. Con el objetivo de adecuar al mercado la formación universitaria en el ámbito audiovisual, parece necesario tener en cuenta las siguientes conclusiones:

La entrada en vigor del Espacio Europeo de Educación Superior (EEES) alteró la estructura de los títulos universitarios, obligados a establecer una conexión más efectiva con el mercado laboral. En este sentido, los estudios de Comunicación Audiovisual, irremediablemente ligados a los cambios tecnológicos, precisan de una permanente y crítica actualización en sintonía con el nuevo panorama digital.

La consolidación de un escenario en el que cada vez alcanzan más relevancia el conocimiento de macrodatos, el diseño de algoritmos y el aprendizaje robótico, unidos a la producción transmedia y una difusión multisoporte, exigen la revisión de las competencias profesionales del comunicador audiovisual, que ahora han de combinar las clásicas destrezas creativas y productivas con otras pertenecientes a nuevos ámbitos de especialización.

Los nuevos perfiles profesionales conviven hoy con los roles tradicionales, si bien a estos últimos les está condicionando la normalización del ecosistema digital. Por el momento se percibe una cierta complementariedad entre creadores y narradores audiovisuales que ya trabajaban en medios analógicos con quienes proceden de ámbitos más técnicos (programadores o desarrolladores). 
Queda clara también la necesidad de que los proyectos audiovisuales se conciban sobre estrategias de comercialización ligadas a las redes sociales y a la proyección transmedia, para lo cual se entienden imprescindibles las competencias en los ámbitos del Marketing Digital y el Social Media.

La introducción de tecnologías y soportes de difusión basadas en interfaces de voz y el cada vez mayor alcance del audio digital en términos creativos y publicitarios -gracias al crecimiento y popularización del formato podcast-aconseja conceder también una mayor presencia a las competencias sonoras en los planes de estudio de Comunicación Audiovisual.

A modo de síntesis, las tres grandes áreas profesionales que se han desarrollado a partir de la implantación del ecosistema digital son: a) el diseño, la programación y el desarrollo de aplicaciones y softwares; b) el marketing digital y la gestión de plataformas de audio y video orientadas -sobre todo- a la explotación online; y c) los efectos visuales FX, la escenografía virtual y el diseño de personajes, escenarios y atmósferas 3D.

Si bien este estudio precisa de continuidad para abordar de manera más detallada cómo incorporar las nuevas competencias profesionales a los planes de estudio, cabe afirmar que los futuros titulados en Comunicación Audiovisual han de acreditar en el mercado su capacidad para adaptarse a los cambios imponen que las tecnologías y los nuevos hábitos de consumo, su dominio sobre las narrativas que posibilita el actual escenario y su visión de conjunto sobre los nuevos modelos de negocio digital. Parece por tanto inaplazable la reformulación de los actuales planes de estudio de los títulos universitarios de Grado en Comunicación Audiovisual, llamados a incorporar un conjunto de materias que permitan adquirir estas nuevas competencias profesionales a la vez que mantener, e incluso tratar de impulsar, las destrezas y saberes básicos propios de las Humanidades.

\section{Referencias bibliográficas}

AEDE (2016): “España, el país con más smartphones por habitante del mundo”, AEDE. Recuperado de http://www.aede.es/ espana-smartphones/: [Consultado el 01/09/2018].

Asociación Española de Radio Online (2017): Primer Directorio Certificado de Radio Online en España 2017. AERO.

ANECA (2005): Libro Blanco de los Títulos de Grado en Comunicación. Barcelona: ANECA.

Ayuntamiento de Madrid (2010): Mesa sectorial de formación para el empleo del sector audiovisual. Barómetro de Empleo de la ciudad de Madrid.

Alberich, J.; Guarinos, V. y Mañas, S. (2009): “Adaptación al EEES del Título de Grado en Comunicación Audiovisual y sinergias modulares comunes en las universidades públicas andaluzas", Icono 14, n. 14, pp. 113-130.

Bandrés, E., García, J.A., Pérez, G. y Pérez, J. (2004): El periodismo en la televisión digital. Barcelona: Paidós.

Bartolomé, A. y Aiello, M. (2006): "Nuevas tecnologías y necesidades formativas. Blended Learning y nuevos perfiles en comunicación audiovisual”, Telos, n. 67, pp. 59-67. 
Besalú-Casademont, R.; Schena, J. y Sánchez-Sánchez, C. (2017): “Competencias más relevantes en los estudios de Comunicación Audiovisual. Las percepciones de profesionales, docentes y estudiantes”, Revista Latina de Comunicación Social, n. 72, pp. 1.536-1.553.

Canales, M. y Peinado, A. (1995): “Grupos de discusión”, en Delgado, J.M. y Gutiérrez, J. (eds.): Métodos y Técnicas cualitativas de investigación en Ciencias Sociales. Madrid: Síntesis, pp. 288-311.

Canavilhas, J. (2013): “Modelos informativos para aparatos móviles: información hipermultimediática y personalizada”, en Colle, R. (coord.): Hacia el periodismo móvil. Alicante: Universitat d'Alacant, Revista Mediterránea de Comunicación, pp. 20-32.

Civil i Serra, M. (2018): "Los estudios universitarios en comunicación en España (2017). Análisis de la situación actual para un debate abierto sobre su futuro estratégico", en Postigo, I. y Recoder, M. (coords.): Los y las «tics» en los estudios de comunicación. Málaga: Asociación Española de Universidades con Titulaciones de Información y Comunicación (ATIC), pp. 15-83.

Cisco (2017): Global Mobile Data Traffic Forecast Update, 2016-2021.

Comisión Nacional de los Mercados y la Competencia (2017): Informe Económico Sectorial de las Telecomunicaciones y el Audiovisual. Madrid: CNMC.

Díaz Nosty, B. (coord.) (2017): Diez años que cambiaron los medios (2007-2017). Barcelona: Ariel y Fundación Telefónica.

Flores, C. (2018): "Netflix invertirá 8.000 millones de dólares en contenido propio para atraer (y mantener) a la audiencia”, $E l$ Economista. Recuperado de https://www.eleconomista.es/tecnologia/noticias/8971126/02/18/Netflix-invertira-8000-millones-de-dolares-en-contenido-propio-para-atraer-a-la-audiencia.html: [Consultado el 16/09/2018].

El Periódico (2018): “Las plataformas de tele rozarán los 800 millones de abonados en el 2023", El Periódico. Recuperado de https://www.elperiodico.com/es/tele/20181001/plataformas-tele-rozaran-800-millones-abonados-2023-7064314: [Consultado el 01/10/2018].

Fernández, J.L. (2014): “La radio en tiempos de movilidad y networking”. Líbero, São Paulo, v. 17, n. 34, pp. 65-76.

Fundación Telefónica (2018): La Sociedad Digital en España 2017. Madrid: Ariel.

Guarinos, V. y Sedeño, A.M. (2013): Narrativas audiovisuales digitales: convergencia de medios, multiculturalidad y transmedia. Madrid: Fragua.

Heredero, O. y Reyes, F. (2016): “Los efectos profesionales de la crisis de la televisión pública española: el creador audiovisual low cost y las nuevas tecnologías”. AdComunica. Revista Científica de Estrategias, Tendencias e Innovación en Comunicación, n. 11, pp. 95-118.

International Federation of the Phonographic Industry (IFPI) (2018): Global Music Report 2018. Annual State of the Industry. Interactive Advertising Bureau (IAB) and PricewaterhouseCoopers (PwC) (2018): Full Year 2017 Podcast Ad Revenue Study: An Analysis of the US Podcast Advertising Industry. 
Jones, D. (1998): “Investigación sobre comunicación en España: Evolución y perspectivas”. ZER: Revista de estudios de comunicación, v. 3, nº 5 .

Miguel, B. (2018): Las competencias digitales en los Grados en Periodismo, Publicidad y Relaciones Públicas y Comunicación Audiovisual en la universidad. El caso de la Comunidad de Madrid. Tesis doctoral, Universidad Complutense de Madrid.

Navío Navarro, M.; González-Díez, L. y Puebla-Martínez, B. (2018): "La formación para la gestión de las redes sociales en los grados de comunicación en España y su adecuación a las competencias demandadas por las organizaciones”. Doxa, n. 26, pp. 127-143.

Orrantia, A. (2018): "La triple secuencia multiplataforma y la audiencia social en COPE. La narración distribuida de contenidos en la antena, la web, redes sociales y alertas a teléfono móvil durante el Día de la Radio en Herrera en COPE'. Hipertext.net, n. 16, pp. 43-50.

Ortiz Sobrino, M. A. (2012): “La reconversión de la formación en comunicación desde el EEES”. Icono14, n. 9, pp. 35-49.

Pedrero, L.M. y Herrera, S, (2017): La notificación push como estrategia informativa de la radio en el entorno digital. El Profesional de la Información, v. 26, n. 6, pp. 1100-1107.

Perlado, M. y Rubio, J. (2012): El comunicador del siglo XXI. Las capacidades que demandan hoy los empleadores y su relación con las titulaciones universitarias. IV Congreso Internacional Latina de Comunicación Social, Universidad de La Laguna.

Perlado, M. y Saavedra, M. (2017): Los estudios universitarios especializados en Comunicación en España. Barcelona: UOC.

Rubio, J. y Perlado, M. (2015): "El fenómeno WhatsApp en el contexto de la comunicación personal: una aproximación a través de los jóvenes universitarios". Icono14, n. 13(2), pp. 73-94.

Siemens, G. (2008): Learning and knowing in Networks: Changing Roles for educators and designers. ITFORUM for discussion. Sierra, J. (coord.) (2010): Los estudios de Ciencias de la Comunicación en el EEES. Madrid: Fragua.

Sierra, J. y Cabezuelo, F. (coord.) (2010): Competencias y perfiles profesionales en los estudios de Ciencias de la Comunicación. Madrid: Fragua. 
